\title{
The Gate Voltage Control of Long DNA Coherent Transport on Insulator Surface
}

\author{
Zhi-Jie Qin ${ }^{1}$ Le Wang ${ }^{1}$, and Gui-Ping Zhang ${ }^{2}$ \\ ${ }^{1}$ School of Physics and Engineering, Zhengzhou University, Zhengzhou, 450001, China and \\ ${ }^{2}$ Department of Physics, Renmin University of China, Beijing 100872, China
}

(Dated: September 27, 2021)

\begin{abstract}
We investigate the coherent transport properties of a DNA chain on a substrate which is subjected to a uniform electric field perpendicular to the surface. On the basis of the effective tight-binding model which simulates charge transport through DNA, the transmission coefficient, Lyapunov exponent, and localization length are numerically calculated by using the transfer-matrix method. It is found that an isolated extended state may appear at the Fermi level for a certain gate voltage when the interaction strength between the chain and the substrate is position dependent but independent of the base-pair sequence, leading to the gate voltage induced Metal-insulator transition (MIT). Moreover, conductance and current-voltage characteristics are also calculated. The relationship of Lyapunov exponent distribution to the current-voltage characteristics is discussed. Two different conduction mechanisms are proposed depending on effectively delocalized states and isolated extended states, respectively. These results may provide perspectives for experimental work aimed at controlling charge transport through DNA-based nanodevices.
\end{abstract}

\section{INTRODUCTION}

Since Eley and Spivey [1] predicted that DNA chain could be conductive, various ingenious experiments have been designed to measure the conductance of DNA. Some of them assert that DNA is an insulator [2, 3, but others show the semiconductor or conductor properties [4 6], and even find the evidence of its superconductor behavior [7]. Although controversy remains for these consequences, it is believed that DNA molecule has the potential for conducting, whose conductivity not only depends on its nature, e.g. the sequence, the sugar-phosphate backbone, and the helix configuration, but also suffers crucial impacts of the environment, e.g. temperature, humidity, solvent, ions, substrate and external field [8, 9]. In 2001, K. H. Yoo et.al have already found in their experiment that the current though DNA can be altered by the gate voltage, that is, DNA molecule is analogous to the field effect transistor(FET) [10]. This phenomenon has reappeared several times in the later experiments 1114]. Theoretically, A.V. Malyshev attributes this phenomenon to the impact of the external field on the helix structure 15. In his model, the DNA chain is suspended without any substrate. However, DNA chains are usually deposited on the surface of some substrate in most of the experiments. In fact, several groups have observed the strong interaction between the mica/SiO2 surface and the DNA chain on it, which does have significant impact on the charge transport through DNA 16 20]. So the study of disorder and substrate effect in one-dimensional DNA chain is still an engaging topic. In fact, with the coexistence of the substrate, gate voltage, and disorder, a complicated situation may appear in DNA chain.

In the present paper, we consider a onedimensional(1D) tight-binding model to investigate

*Electronic address: qin'zhijie@163.com the combined effect of disorder, the interaction between DNA chain and substrate, and the gate voltage. The disorder is introduced by random distribution of $\operatorname{poly}(\mathrm{dA})-\operatorname{poly}(\mathrm{dT})$ and poly $(\mathrm{dG})-\operatorname{poly}(\mathrm{dC})$ in DNA chain. An isolated extended state has been founded for a certain gate voltage when the interaction strength is dependent on the position of chain but independent of the base-pair sequence, which leads to the gate voltage induced Metal-insulator transition (MIT). The dependance of the transmission spectrum and Lyapunov exponent(LE) on the energy, the gate voltage and chain length are calculated. The associated behavior is studied and the relationship of the results to the distribution of Lyapunov exponent is also discussed.

The paper is organized as follows: In the next section we describe the basic formalism in our calculations. In section 3 we present the main results and discuss the related physical implication. The last section is devoted to a brief summary of conclusions.

\section{THE BASIC FORMALISM}

We consider a 1D DNA chain with interaction between chain and substrate as illustrated in Fig. 1. In the tightbinding approximation, the Hamiltonian is described as [21]

$$
\begin{aligned}
H= & \sum_{n}\left[\epsilon_{n} c_{n}^{\dagger} c_{n}-t_{n}\left(c_{n}^{\dagger} c_{n+1}+H . c .\right)\right] \\
& +\sum_{n=1}^{N}\left[\left(\epsilon_{b n}+V_{g}\right) b_{n}^{\dagger} b_{n}-t_{b n}\left(c_{n}^{\dagger} b_{n}+H . c .\right)\right],
\end{aligned}
$$

where each lattice site represents a poly $(\mathrm{dA})$-poly $(\mathrm{dT})$ base or poly $(\mathrm{dG})$-poly $(\mathrm{dC})$ base for $n \in[1, N]$. The operator $c_{n}^{\dagger}\left(c_{n}\right)$ creates(annihilates) a charge at the $n$th site of the base pair in the DNA chain $(1 \leq n \leq N)$, of the left electrode $(n \leq 0)$, of the right electrode $(n \geq N+1)$. 
The operator $b_{n}^{\dagger}\left(b_{n}\right)$ creates(annihilates) a charge at the $n$th site of the substrate $(1 \leq n \leq N)$. $\epsilon_{n}$ and $t_{n}$, respectively, denote the site energy and hopping integral in DNA chain, $\epsilon_{b n}$ is the site energy in the substrate, $t_{b n}$ stands for coupling between chain and substrate, and $V_{g}$ is the gate-voltage.

On each substrate site there is a highly localized orbit formed via the interaction of the base pair, the sugarphosphate structure and the substrate surface. So, it is reasonable to consider that different base pairs corresponds to different values of coupling strength $t_{b n}$ and side site energy $\epsilon_{b n}$, that is, for base pair $G C$, we have $\epsilon_{b n}=\epsilon_{b G C}, t_{b n}=t_{b G C}$; for base pair $A T$, we have $\epsilon_{b n}=\epsilon_{b A T}, t_{b n}=t_{b A T}$. Obviously, this is a kind of longitudinal short-range correlation analogous to a layer structure that may produce a band of extended states 22.].

If the base pair GC (or AT) is distributed randomly and independently with a probability $p($ or $1-p)$, the disorder strength $\mathrm{W}$ will depend on $p$ and $\left|\epsilon_{A T}-\epsilon_{G C}\right|$. For $p=1$ or 0 cases, we have a period chain with two energy bands, the highest occupied molecular orbital (HOMO) and the lowest unoccupied molecular orbital (LUMO). For a fixed $\left|\epsilon_{A T}-\epsilon_{G C}\right| \neq 0$ case, the most disordered state is given by $p=0.5$ [22] and in this case the transmittivity of the LUMO is almost zero. So the following text only focuses on the HOMO with $p=0.5$.

In the site representation, the Schrödinger equation $H|\psi\rangle=E|\psi\rangle$ corresponding to Eq. (1) becomes

$$
\begin{array}{r}
\left(E-\epsilon_{n}\right) \psi_{D, n}=-t_{n-1} \psi_{D, n-1}-t_{n} \psi_{D, n+1}-t_{b n} \psi_{S, n} \\
\left(E-\epsilon_{b n}-V_{g}\right) \psi_{S, n}=-t_{b n} \psi_{D, n} .
\end{array}
$$

Here, $\psi_{D, n}$ is the amplitude of the wave function at the $n$th site of the chain(the $n$th base pair site) and $\psi_{S, n}$ is the amplitude of the wave function at the $n$th site of the substrate. After the decimation of $\psi_{S, n}[23,24]$, the corresponding Schrödinger equation of the Hamiltonian (1) is

$$
\left(\epsilon_{n}^{\prime}-E\right) \psi_{D, n}=t_{n-1} \psi_{D, n-1}+t_{n} \psi_{D, n+1} .
$$

Here, $\epsilon_{n}^{\prime}$ is the renormalized on site energy, which is defined as

$$
\epsilon_{n}^{\prime}=\epsilon_{n}+\frac{t_{b n}^{2}}{E-\left(\epsilon_{b n}+V_{g}\right)} .
$$

The renormalized site energy $\epsilon^{\prime}$ includes the influence of substrate on conduction state in DNA chain, and it is a function of the gate voltage $V_{g}$ and the incident electron energy $E[15]$.

The Eq. (3) can be rewritten as relations between coefficient of adjacent pints in the form of the transfer matrix

$$
\left(\begin{array}{c}
\psi_{D, n+1} \\
\psi_{D, n}
\end{array}\right)=T_{n}\left(\begin{array}{c}
\psi_{D, n} \\
\psi_{D, n-1}
\end{array}\right)
$$

with

$$
T_{n}=\left(\begin{array}{cc}
\frac{\left(\epsilon_{n}^{\prime}-E\right)}{t_{n}} & -\frac{t_{n-1}}{t_{n}} \\
1 & 0
\end{array}\right) .
$$

For a 1-D chain with length $L$, the coefficients at one end are related to the coefficients at the other end with the transfer matrices

$$
\left(\begin{array}{c}
\psi_{L} \\
\psi_{L-1}
\end{array}\right)=\left(\prod_{j=1}^{L-1} T_{L-j}\right)\left(\begin{array}{c}
\psi_{1} \\
\psi_{0}
\end{array}\right) .
$$

The Lyapunov exponents(LE) are the logarithms of eigenvalues of the transfer matrix, and can be calculated by using the transfer-matrix method, in which the orthonormalization procedure is adopted [25].

In this paper, the transmittivity [26] and the localization length (LL) 26, 27] which is the inverse of the LE are calculated with the transfer matrix (TM). The current is computed with the Landauer-Büttiker formula

$$
I=\frac{2 e}{h} \int_{-\infty}^{+\infty} T(E)\left(f_{L}-f_{R}\right) d E
$$

here $f_{L / R}(E)=\left\{1+\exp \left[\left(E \pm e V_{d} / 2-E_{F}\right) / k_{B} T\right]\right\}^{-1}$ is the Fermi distribution on the left/right lead. $E_{F}$ and $V_{d}$ are the equilibrium Fermi energy and applied voltage, respectively. $T(E)$ is the transmission through $\mathrm{N}$ base pairs.

\section{NUMERICAL RESULTS}

In the numerical calculations we adopt a DNA chain with different length $N$. The lattice structure is illustrated in Fig. 1. The values of the parameters are chosen as the following [9]: $\epsilon_{n}$ is the average of the complementary bases energies, $\epsilon_{G C}=\left(\epsilon_{G}+\epsilon_{C}\right) / 2$, $\epsilon_{A T}=\left(\epsilon_{A}+\epsilon_{T}\right) / 2[28]$. Here, $\epsilon_{G}=7.75 \mathrm{eV}, \epsilon_{C}=8.87 \mathrm{eV}$, $\epsilon_{A}=8.24 \mathrm{eV}, \epsilon_{T}=9.14 \mathrm{eV}$. The site energy on the lead $\epsilon_{m}=5.36$ is the work function of platinum. The site energy $\epsilon_{b G C}=10.1 \mathrm{eV}$ and $\epsilon_{b A T}=11 \mathrm{eV}$ are slightly higher than the base pair energy $\epsilon_{n}$. For simplicity, both the hopping integral on the DNA and at the contacts are fixed at $0.4 \mathrm{eV}$, to minimize the contact scattering [29]. The hopping integral on the leads is $12 \mathrm{eV}$. The surface coupling parameters $t_{b G C}=4.4 \mathrm{eV}$ and $t_{b A T}=5.0 \mathrm{eV}$ which are stronger than the usual coupling between base pair and sugar-phosphate structure [16, 18]. The latter is about $1.0 \mathrm{eV}[9,30]$ and is neglected here. Fermi energy is set to be $5.36 \mathrm{eV}$ through out the paper.

First, we present the results for a DNA chain with different length $N$. Figure 2 shows the rescaled localization length(RLL) $\Lambda / N$ as a function of chain length $N$ with different gate voltage $V_{g}$ at Fermi energy. Here, $\Lambda$ represents the localization length. It can be seen, for most values of $V_{g}$, that the $\Lambda / N$ decreases with increasing 


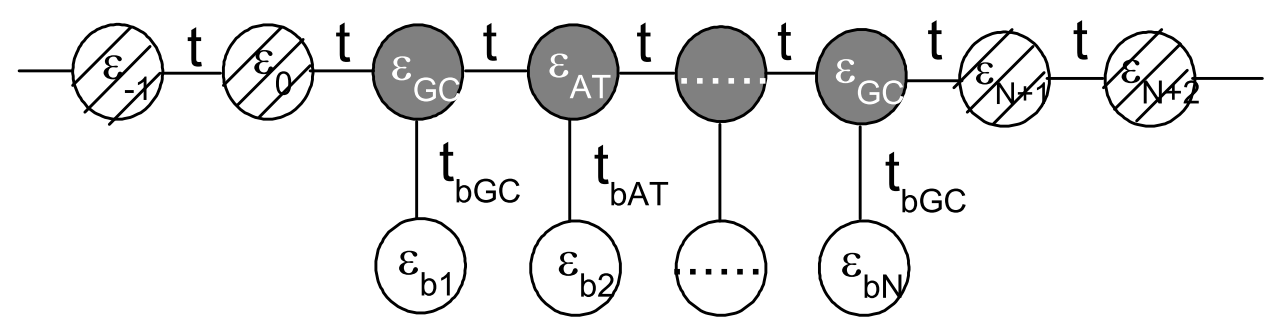

FIG. 1: Schematic illustration of extending a DNA chain with substrate to a very long chain. The black circles represent the DNA chain, white circles denote substrate, and shaded circles stand for the attached leads. Electronic pathways are shown as lines.

$N$, while, for some given gate voltages $V_{g 1} \sim 0.57 \mathrm{~V}$ and $V_{g 2} \sim 3.88 \mathrm{~V}, \Lambda / N$ is slightly increased with $N$. The RLL $\Lambda / N$ decays with power rate as increasing $\mathrm{N}$ for localized state, while it fluctuates throughout for extended state [31, 32]. This difference in scaling behavior indicates that there may exist delocalized states for given gate-voltages in the thermodynamic limit.

In Fig. 3(a), we plot the Lyapunov exponent as a function of the energy $E$ with different gate voltage $V_{g}$ for $N=10^{6}$. It can be seen, for all the investigated values of $V_{g}$, that the smallest Lyapunov exponent always lies in a narrow region of energy and appears at different energy $E$. Since the transport properties of the system mainly depends on the quasi-particle's states near the Fermi levels, Fig. 3(b) shows Lyapunov exponent as a function of the gate voltage $V_{g}$ at Fermi energy. It can be seen that there exist two valley-like structures at $V_{g}=V_{g 1} \sim 0.57$ and $V_{g}=V_{g 2} \sim 3.88$, which are exactly the same gate voltages that corresponds to the delocalization behaviors in Fig. 2. This valley-like structure indicates that there may exist a delocalization-localization translation around the valleys. Note that only the points at the bottom of the valleys correspond to the smallest Lyapunov exponent. Combined the results of Fig. 3(a) and 3(b), we could see, at the bottom of the valleys, i.e. $V_{g 1}$ and $V_{g 2}$, that the narrow energy region which corresponds to the smallest Lyapunov exponent appears around Fermi levels. Considering the scaling behavior of the RLL in Fig. 2, we could conclude that these states, which correspond to the smallest Lyapunov exponent at $V_{g}=V_{g 1}$ and $V_{g}=V_{g 2}$ in Fig. 3(b), are isolated extended states at Fermi energy.

In order to understand the origin of these isolated extended states, Eq. (3) should be discussed further. Since only two kinds of base pairs G-C and A-T are considered in present system, there are two kinds of $\epsilon_{n}^{\prime}$, i.e. $\epsilon_{A T}^{\prime}$ and $\epsilon_{G C}^{\prime}$. If parameters satisfy the following conditions,

$$
\begin{gathered}
\epsilon_{A T}^{\prime}=\epsilon_{G C}^{\prime}=\epsilon^{\prime}, \\
E \in\left[\epsilon^{\prime}-2 t, \epsilon^{\prime}+2 t\right],
\end{gathered}
$$




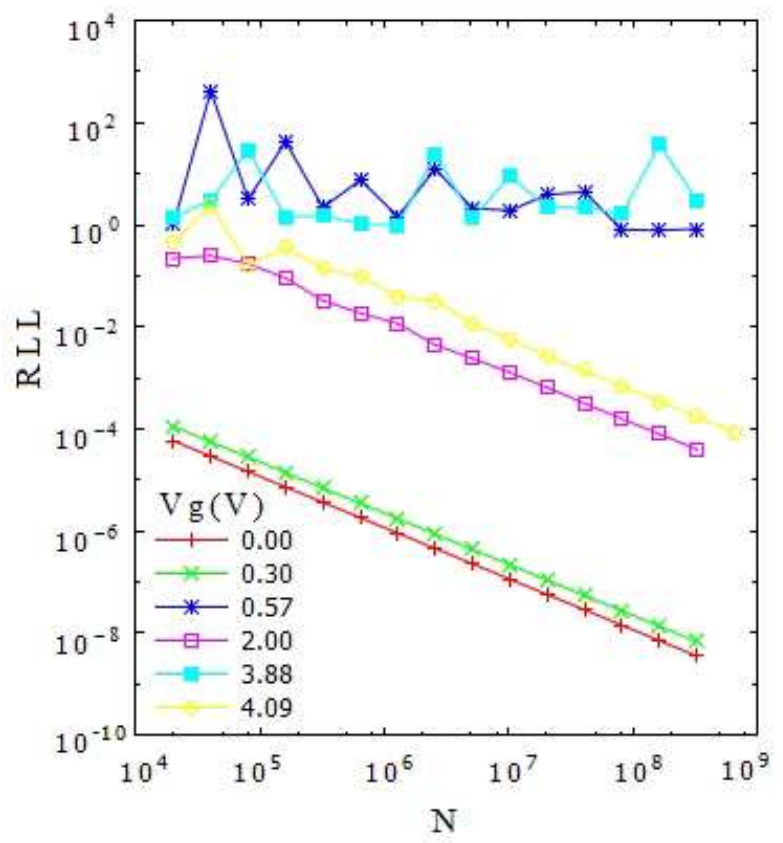

FIG. 2: (Color online.) The rescaled localization length (RLL) as a function of chain length $N$ at $E=E_{F}$; red line for $V_{g}=0.00 \mathrm{~V}$, green line for $V_{g}=0.30 \mathrm{~V}$, blue line for $V_{g}=V_{g 1} \approx 0.57 \mathrm{~V}$; pink line for $V_{g}=2.00 \mathrm{~V}$, light blue line for $V_{g}=V_{g 2} \approx 3.88 \mathrm{~V}$; yellow line for $V_{g}=4.09 \mathrm{~V}$.

then eq. (3) becomes

$$
\left(\epsilon^{\prime}-E\right) \psi_{D, n}=t \psi_{D, n-1}+t \psi_{D, n+1}
$$

which corresponds to an Schrödinger equation for a periodic chain. In other words, the solution of the above conditions corresponds an extended state with energy $E$. So Eq. (8) and (9) are a criteria for finding an isolated extended states within our model. The origin of this extended state comes from the constructive interference between DNA chain sites and substrate sites [33]. Condition for Eq. (9) ensures $\mathrm{E}$ in the propagation band of the renormalized period chain with onsite energy $\epsilon^{\prime}$. Here, $\epsilon^{\prime}$ varies with $\mathrm{E}$ according to Eq. (8). Both Eq. (8) and (9) are independent of GC concentration $p$ and base pair arrangements, which indicates that the existence of the extended state will not be impacted by specific base pair sequences. This mechanism is similar to the cases in refs. 22, 34].

Substitute the renormalized on site energy $\epsilon^{\prime}$ in eq. (4) into the above criteria for isolated extended states, Eq. (8) and (9) turn into the following forms:

$$
\begin{gathered}
E-e V_{g}=-\frac{\alpha}{2} \pm \sqrt{\gamma},(\gamma \geq 0) . \\
E \in\left[\epsilon_{+}^{\prime}-2 t, \epsilon_{+}^{\prime}+2 t\right] \cup\left[\epsilon_{-}^{\prime}-2 t, \epsilon_{-}^{\prime}+2 t\right] .
\end{gathered}
$$

Here, $\triangle \epsilon=\epsilon_{A T}-\epsilon_{G C}, \alpha=\Delta \epsilon^{-1}\left(t_{b A T}^{2}-t_{b G C}^{2}\right)-\left(\epsilon_{b A T}+\right.$ $\left.\epsilon_{b G C}\right), \beta=\epsilon_{b A T} \epsilon_{b G C}\left[\triangle \epsilon^{-1}\left(t_{b A T}^{2} \epsilon_{b A T}^{-1}-t_{b G C}^{2} \epsilon_{b G C}^{-1}\right)-1\right]$,
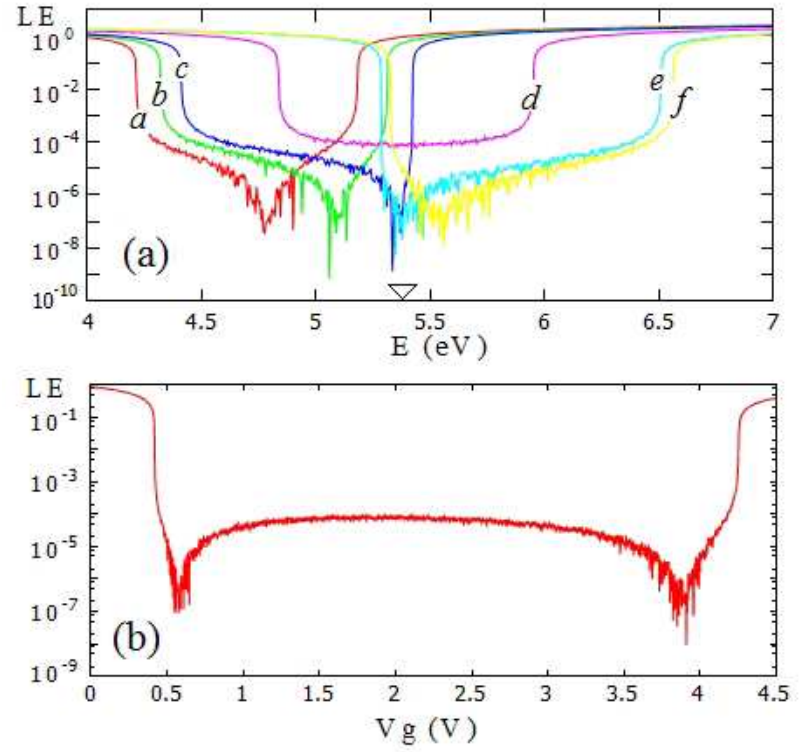

FIG. 3: (Color online.) (a) Lyapunov Exponent(LE) as a function of energy $\mathrm{E}$ with different $V_{g}$; curve a, $V_{g}=0 \mathrm{~V}$; $\mathrm{b}, V_{g}=0.30 \mathrm{~V} ; \mathrm{c}, V_{g}=V_{g 1} \approx 0.57 \mathrm{~V} ; \mathrm{d}, V_{g}=2.00 \mathrm{~V} ; \mathrm{e}$, $V_{g}=V_{g 2} \approx 3.88 \mathrm{~V} ; \mathrm{f}, V_{g}=4.09 \mathrm{~V}$. The open triangle marks $E_{F}=5.36 \mathrm{eV}$. (b) LE versus $V_{g}$ at $E_{F}$. The DNA length $\mathrm{N}$ is $10^{6}$.

$\gamma=\beta+\alpha^{2} / 4$, and $\epsilon_{ \pm}^{\prime}=\epsilon_{A T}+t_{b A T}^{2} /\left(-\alpha / 2 \pm \sqrt{\gamma}-\epsilon_{b A T}\right)$. Therefore, the relation of the isolated extended state energy $\mathrm{E}$ and the gate voltage $V_{g}$ is two pieces of paralleled line segments with slope $e$ and with the terminal vertexes depending on site energies and hopping integrals. When $E=E_{F}=5.36 \mathrm{eV}$, Eq. (10) gives two solutions for $V_{g}$ : $V_{g} \approx 0.57\left(=V_{g 1}\right)$ and $V_{g} \approx 3.88\left(=V_{g 2}\right)$, which means, for these two cases, that there is an isolated extended state at Fermi level $E_{F}$. These results are consistent with the previous results in Fig. (2) and Fig. (3).

Figure 4 shows the transmission coefficients as a function of energy for different $V_{g}$ and $N$. It can be seen that there is a transmission band for the chain with different gate voltages when the chain length is short. With the increase of chain length $N$, some transmission band would disappear, i.e. for $V_{g}=2$ case, while others may leave a sharp peak around the value of energy of the isolated extended state, i.e. for $V_{g}=0, V_{g 1}$, and $V_{g 2}$ cases. This implies that there are two kinds of mechanisms for transmission. The former depends on the effectively delocalized states 35], which forms in a system shorter than its localization length, while the latter depends on an isolated extended state. With the increase of system length, the effectively delocalized states disappear, however, the isolated extended state is still there. In other words, with the increase of $\mathrm{N}$, the transmission band decays but leaves a sharp peak around the energy of an isolated extended state for $V_{g}=0, V_{g 1}$, and $V_{g 2}$ cases, or vanishes for $V_{g}=2$ case.

In Fig. 5, we plot the conductance of chain with differ- 
ent gate voltage as a function of chain length $N$. It can be seen that the conductance $G$ decreases rapidly with the increase of the length $N$ for cases with $V_{g} \neq V_{g 1}$ or $V_{g 2}$. This implies that the number of the effectively delocalized states around Fermi energy for such cases decreases rapidly with the increase of length of the system. It can also be seen that, for $V_{g}=V_{g 1}$ and $V_{g}=V_{g 2}$ cases, the conductance shows a strong oscillation. The oscillation might be caused by the coherent scattering of Bloch's wave at the two contacts of the leads 29]. For $V_{g}=4.085$ case, $G_{0}$ decays exponentially with some oscillation because the Fermi level is close to the isolated extended state. This shows that conductance mainly depends on the chain length $N$ and the energy position of the isolate extent states.
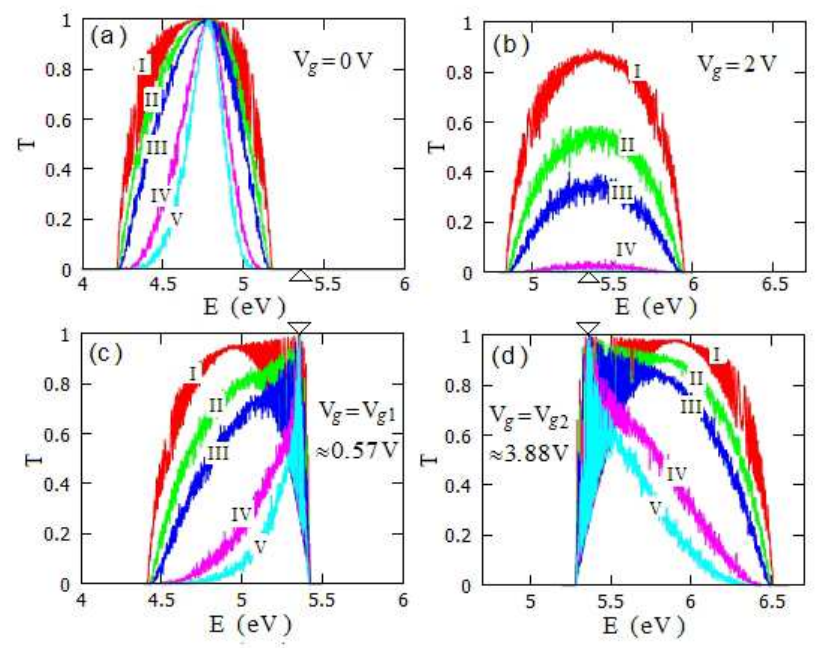

FIG. 4: (Color online.) Transmission coefficient $T(E)$ of a chain with different $V_{g}$ as a function of energy. The curve I, II, III, IV, V represent for the cases with $N=1000, N=$ 5000, $N=10000, N=50000$, and $N=100000$, respectively. The probability $p=0.5$. The results are averaged over 100 samples. The Fermi level $E_{F}=5.36 \mathrm{eV}$ is labelled by the open triangles.

In order to consider the combined effect of the gate voltage and the chain length, in Fig. 6 , we plot $I-V_{d}$ characters of DNA with different length $N$ for different values of gate voltage $V_{g}$. The current $I$ is averaged with 100 realizations. It can be seen, with the increase of the length $N$, that, for some kind of $V_{g}$, the current $I$ decreases and even becomes zero for some certain values of gate voltage, while, for the other kind of $V_{g}$ around $V_{g 1}$ and $V_{g 2}$, the current still exists for the regions of $V_{g}$ around $V_{g 1}$ and $V_{g 2}$ when $N$ is up to $10^{5}$. This shows that transport properties of DNA are suppressed by the length.

The difference between these behaviors of currents lies in different conduction mechanisms and can be understood by the concept of the effective delocalized state 35 . For the short chain with $N \leq \Lambda$ cases, the quasiparticles are in the effectively delocalized states with high

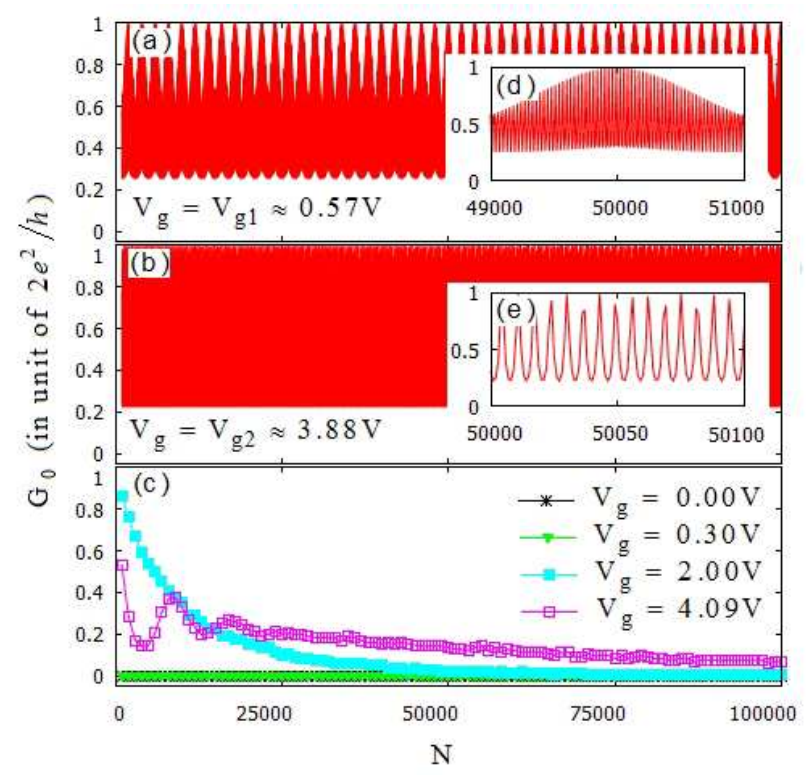

FIG. 5: (Color online.) Conductance $G$ as a function of DNA length $\mathrm{N}$ with (a) $V_{g}=V_{g 1}$, (b) $V_{g}=V_{g 2}$, (c) $V_{g}=0 e V$, $0.30 \mathrm{eV}, 2.00 \mathrm{eV}$, and $4.09 \mathrm{eV}$. The inset Fig. (d) and (e) show the details of the oscillation in figure (a) and (b), respectively. The oscillation are very dense in figure (a) and (b). The results in figure (c) are averaged over 1000 samples.

transmittivity spread all over the band and conceal the impact of the isolated extended state. This situation corresponds to the current peak for the case of $V_{g}=2$ in Fig. 6. With further increases of $\mathrm{N}$, the effectively delocalized states eventually vanish, while the impact of the isolated extended state emerges. Therefore, two current peak bands form respectively around $V_{g 1}$ and $V_{g 2}$ where the isolated extended state lies around the Fermi level. However, the two peaks are not exactly at $V_{g 1}$ and $V_{g 2}$, because the Landauer-Büttiker formula is asymptotically equal to $I=2 e h^{-1} \int_{E_{F}-e V_{d} / 2}^{E_{F}+e V_{d} / 2} T(E) d E$ at low temperature, and the maximum of current does not require the transmission peak exactly at $E_{F}$, but the maximum area under the curve of $T(E)$ in the conducting interval $\left[E_{F}-e V_{d} / 2, E_{F}+e V_{d} / 2\right]$. However, the larger the length $\mathrm{N}$ is, the more proximity to $V_{g 1}$ and $V_{g 2}$ the current peaks get. In other words, the effective delocalized states will vanish with the increase of chain length, while the isolated extended states may still exist.

\section{CONCLUSIONS AND DISCUSSION}

We have investigated the coherent transport properties of a DNA chain with the interaction between DNA and substrate. Using the tight-binding model and transfer matrix technique, it is found that there is an isolated extended state which is robust against the DNA sequence rearrangements. Physically, the origin of such an isolated extended state is the interference between DNA 

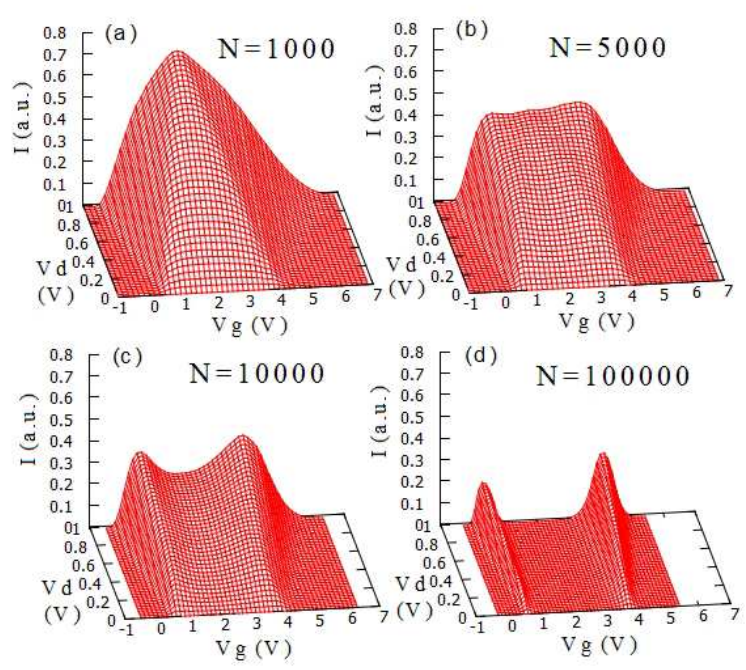

FIG. 6: (Color online.) Total current $I$ of a chain with different length $\mathrm{N}$ as a function of the gate voltage $V_{g}$ and applied voltage $V_{d}$ on the two leads with (a) $N=1000$, (b) $N=5000$, and (c) $N=10000$; (d) $N=100000$. The temperature is 0.1 $\mathrm{K}$. The results are averaged over 100 samples. Other parameters are the same as those in Fig. 2.

chain sites and substrate sites. Based on this mechanism, the controllable long distance coherent transport in mesoscale can be achieved by fabricating single molecular FET with long disordered DNA chains (e.g., $\lambda-D N A$ ) adhering to substrate surface. This mechanism is also valid for the general nanowire of binary alloy or conductive polymer interacting with substrate surface. Furthermore, since the principle of complementary nucleobases is a natural transverse correlation of short range, this mechanism may be generalized to the ladder model of DNA, and may therefore impact the spin selective transport through double strand DNA as well [36], which needs further study of both theory and experiment. The conductance and current-bias characteristics shows that Metal-insulator transition (MIT) could emerge by varying the gate voltage or the chain length. Two different conduction mechanisms attributing to effectively delocalized states and isolated extended states, respectively, are proposed. These results may provide perspectives for experimental work aimed at controlling charge transport through DNA-based nanodevices.

\section{ACKNOWLEDGEMENTS}

We would like to thank Professor S. J. Xiong for many helpful discussions. This work was supported by the National Fund of Natural Sciences of China (Grant No. 11204372) and a grant for STRP from the HNED of China (Grant No. 14B140013).
[1] D. D. Eley and D. I. Spivey, D. D. Eley and D. I. Spivey, Trans. Faraday Soc. 58, 411, (1962)

[2] E. Braun, Y. Eichen, U. Sivan, and G. Ben-Yoseph, Nature (London) 391, 775 (1998)

[3] Y. Zhang, R. H. Austin, J. Kraeft, E.C. Cox, and N. P. Ong, Phys. Rev. Lett. 89, 198102 (2002)

[4] D. Porath, A. Bezryadin, S. de Vries, and C. Dekker, Nature (London) 403, 635 (2000)

[5] H. Cohen, C. Nogues, R. Naaman, and D. Porath, Proc. Natl. Acad. Sci. U.S.A. 102, 11589 (2005)

[6] E. Shapir, H. Cohen, A. Calzolari, C. Cavazzoni, D. A. Ryndyk, G. Cuniberti, A. Kotlyar, R. D. Felice and D. Porath, Nature Mater. 7, 68 (2008)

[7] A. Yu. Kasumov, M. Kociak, S. Guron, B. Reulet, V. T. Volkov, D. V. Klinov, H. Bouchiat, Science 291, 280 (2001)

[8] R. G. Endres, D. L. Cox, and R. R. P. Singh, Rev. Mod. Phys. 76, 195 (2004)

[9] E.L. Albuquerque, U.L. Fulco, V.N. Freire, E.W.S. Caetano, M.L. Lyra, and F.A.B.F. de Moura, Phys. Repo. 535, 139 (2014)

[10] K. H. Yoo, D.H. Ha, J. O. Lee, J.W. Park, J. Kim, J. J. Kim, H. Y. Lee, T. Kawai, and H. Y. Choi, Phys. Rev. Lett. 87, 198102 (2001)

[11] S. Maeno, N. Matsuo, S. Nakamura, A. Heya, T. Takada, K. Yamana, M. Fukuyama, and S. Yokoyama, IEICE Electron. Express 11, 20130900 (2014)
[12] S. Maeno, S. Takagi, N. Matsuo, K. Yamana, A. Heya, T. Takada, and S. Yokoyama, IEEE 10.1109IMFEDK. 2012.6218621 (2012)

[13] S. Takagi, T. Takada, N. Matsuo, S. Yokoyama, M. Nakamura, and K. Yamana, Nanoscale 4, 1975(2012)

[14] S. Roy, H. Vedala, A. D. Roy, D-h. Kim, M. Doud, K. Mathee, H-k. Shin, N. Shimamoto, V. Prasad, and W. Choi, Nano Lett. 8, 26 (2008)

[15] A.V. Malyshev, Phys. Rev. Lett. 98, 096801(2007)

[16] A. Yu. Kasumov, D. V. Klinov, P.-E. Roche, S. Guron, and H. Bouchiat, Appl. Phys. Lett. 84,1007 (2004)

[17] T. Heim, T. Mlin, D. Deresmes, and D. Vuillaume, Appl. Phys. Lett. 85, 2637 (2004)

[18] T. Heim, D. Deresmes, and D. Vuillaume, J. Appl. Phys. 96, 2927 (2004)

[19] J. Bechhoefer and D. Sen, Phys. Rev. Lett. 93,239801(2004)

[20] Y. Zhang, R. H. Austin, E. C. Cox, and N. P. Ong, Phys. Rev. Lett. 93, 239802 (2004)

[21] G. Cuniberti, L. Craco, D. Porath, and C. Dekker, Phys. Rev. B 65, 241314(R)(2002)

[22] A-M. Guo, S-J. Xiong, X. C. Xie , and Q-f. Sun, J.Phys.:Condens.Matter 25, 415501 (2013)

[23] R. Farchioni, G. Grosso, and G. P. Parravicini, Phys. Rev. B 85, 165115 (2012)

[24] G. Xiong, Phys. Rev. B 76, 153303 (2007)

[25] A. MacKinnon, B. Kramer, Phys. Rev. Lett. 47, 1546 
(1981)

[26] Keith Slevin and Yoichi Asada, Phys. Rev. B 70, 054201 (2004)

[27] A. MacKinnon and B. Kramer, Z. Phys. B Condensed Matter 53, 1 (1983)

[28] A-M. Guo, S-J. Xiong, Z. Yang, and H-J. Zhu, Phys. Rev. E 78, 061922 (2008)

[29] Y. Zhu, C. C. Kaun, and H. Guo, Phys. Rev. B 69, $245112(2004)$

[30] E. Maci and S. Roche, Nanotechnology 17, 3002 (2006)

[31] H. Shima, T. Nomura, and T. Nakayama, Phys. Rev. B
70, 075116 (2004)

[32] A-M. Guo and S-J. Xiong, Phys. Rev. B 83, 245108 (2011)

[33] Andrey E. Miroshnichenko* et al, Rev. Mod. Phys. 82, $2257(2010)$

[34] Z-Y. Zhang, S-J. Xiong, and S. N. Evangelou, J. Phys.: Condens. Matter 10, 8049 (1998)

[35] H. C-Nuñez and P. A. Schulz, Phys. Rev. B 78, 235404 (2008)

[36] B Gohler et al. Science 331, 894 (2011) 